\title{
Affine term structure as multi-soliton
}

\author{
Hidemi Aihara $^{1}$, Jirô Akahori ${ }^{1}$ and Edouard Grenier ${ }^{2}$ \\ ${ }^{1}$ Department of Mathematical Sciences, Ritsumeikan University, 1-1-1, Nojihigashi, Kusatsu, \\ Shiga 525-8577, Japan \\ 2 École Nationale Supérieure de Techniques Avancées, 828, Boulevard des Maréchaux, 91762 \\ Palaiseau Cedex \\ E-mail hide.v3v.pooh@gmail.com
}

Received December 6, 2013, Accepted December 25, 2013

\begin{abstract}
In the real market, the term structure of forward rates exhibits some humps. The quadratic Gaussian term structure models or affine term structure models well explain this phenomena. In this research, we give a new insight, where we understand the humps as multi-soliton that are related to $\mathrm{KdV}$ solitons.
\end{abstract}

Keywords term structure of interest rates, humps, affine class, quadratic Gaussian model, solitons

Research Activity Group Mathematical Finance

\section{Introduction}

The spot interest rate $r(t, T)$ is the rate per unit of time (normally it is one year) at which one can (in practice, the rate can vary depending on who they are and how it is agreed but we ignore such credit risks/counter party risks here) borrow (lend) cash at time $t$ and repay (be repaid) at time $T$. Theoretically it is related to the price $P(t, T)$ of the zero-coupon bond maturing at $T$ as

$$
r(t, T)=-\frac{1}{T-t} \log P(t, T) .
$$

In practice, the rate so defined is called zero rate. The function

$$
T \mapsto r(t, T)
$$

is what we call term structure of spot rates, or in practice it is rather function in $x=T-t$;

$$
x \mapsto r(t, t+x),
$$

which is often referred to as yield curve.

In theoretical finance, one rather work on the term structure of (the instantaneous) forward rates, which is given by

$$
T \mapsto f(t, T)=-\frac{\partial}{\partial T} \log P(t, T),
$$

or

$$
x \mapsto f(t, t+x)=-\left.\frac{\partial}{\partial T} \log P(t, T)\right|_{T=t+x} .
$$

This is because the forward rate is easier to handle mathematically. In particular to impose arbitrage-free property to the term structure.

In real market, however, the term structure of spot rates behaves nicer. According to the series of studies by N.L.Liu and her collaborators [1-3], from the term structure of spot rates only two or three factors up to almost $99 \%$ are detected when applied a principal component analysis (or its variants), while that of forward

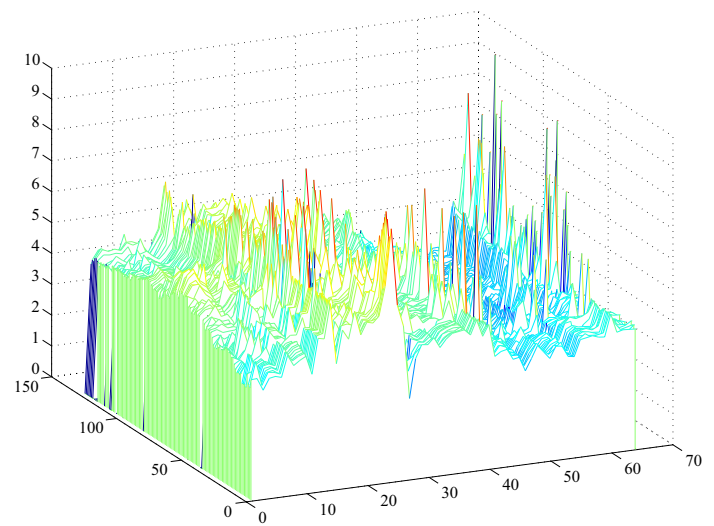

Fig. 1. Typical forward rate movement: EU zero rate.

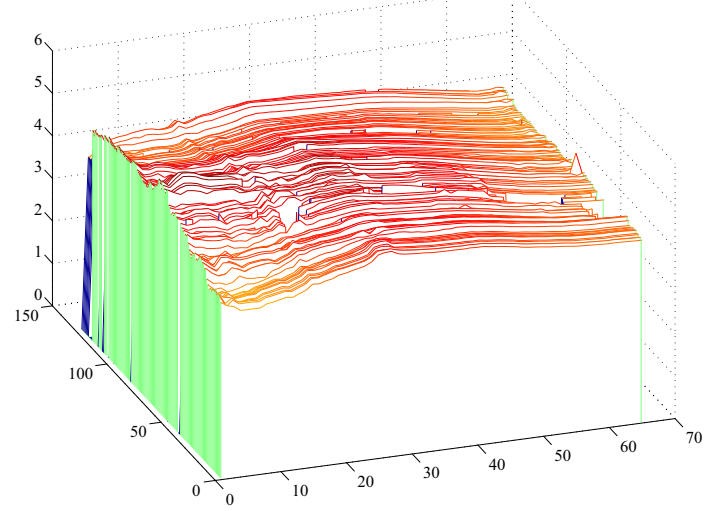

Fig. 2. Spot rate movement of the same data as Fig. 1.

rates exhibits more than 10 , sometimes 15 , or even more factors. Much more straightforward peculiarity is that the samples of the term structure of forward rates often have more humps than those of spot rates.

The main aim of the present paper is to propose a new point of view where the humps are understood as a kind of solitons.

The rest of the paper is organized as follows. In Section 
2 , we illustrate our idea by a primitive one dimensional example. In Section 2.1, we present a brief introduction to solitons. In Section 3, we give a multi-dimensional version of the observation made in Section 2. We emphasize that a class of affine (quadratic Gaussian) models exhibits multi-soliton shape term structures. Finally in Section 4, we remark that the solitons appearing in the term structure models are related to a non-linear partial differential equations called $K d V$ equations.

\section{A primitive example} Let

To explain the idea, we start with a primitive example.

$$
P(t, T)=\mathbf{E}\left[e^{-\frac{1}{2} \int_{t}^{T} c^{2}\left|W_{s}\right|^{2} d s} \mid W_{t}\right], \quad 0 \leq t \leq T,
$$

where $W$ is a 1-dimensional Brownian motion. This formula defines an arbitrage-free bond market, which is a simplest example of the quadratic Gaussian model, and at the same time, an affine term structure model (see e.g. [4]) where we consider $|W|^{2}$ to be a state variable. In fact, we have an explicit expression as

$$
P(t, T)=\left(\cosh (c(T-t))^{-\frac{1}{2}} e^{-\frac{c}{2} \tanh (c(T-t))\left|W_{t}\right|^{2}},\right.
$$

and the (instantaneous) forward rate $f(t, T)=-\partial_{T}$ $\log P(t, T)$ is then expressed as

$$
\begin{aligned}
f(t, T)=\frac{c}{2} & \tanh (c(T-t)) \\
& +\frac{c^{2}\left|W_{t}\right|^{2}}{2} \operatorname{sech}^{2}(c(T-t)),
\end{aligned}
$$

which is an affine function in the state variable.

By (1), we know that

$$
T \mapsto-\log P(t, T)
$$

is increasing, and therefore the term structure of spot rates under this model behaves nicely, while one notices that

$$
T \mapsto f(t, T)
$$

is a rational function of $e^{c(T-t)}$ and $e^{-c(T-t)}$, which is, what we will call in local terminology, a soliton.

Fig. 3 exemplifies a sample path of the affine forward rate.

\subsection{Solitons}

In general, a traveling wave solution to a non-linear (evolution-type) differential equation is not stable; it collapses from the top. The soliton solutions are exceptions. They have (sometimes more than two) solitary waves=humps, and the humps are quite stable even after the "collisions". Somehow they behave like particles, and that is why they are called "solitons", which are shown in Fig. 4. Mathematically, solitons can be defined as some rational functions of exponentials (see [5]). More precisely, it is something like

$$
u(t, x)=\frac{f}{g}=\frac{\sum_{i} K_{i} e^{A_{i} t-B_{i} x}}{\sum_{i} L_{i} e^{C_{i} t-D_{i} x}},
$$

where $A_{i}, B_{i}, C_{i}, D_{i}, K_{i}$ and $L_{i}$ are constants, and the summations are finite ones. Here we assume $\max _{i} C_{i} \geq$ $\max _{i} A_{i}$ and $\min _{i} C_{i} \leq \min _{i} A_{i}$ to ensure the existence of

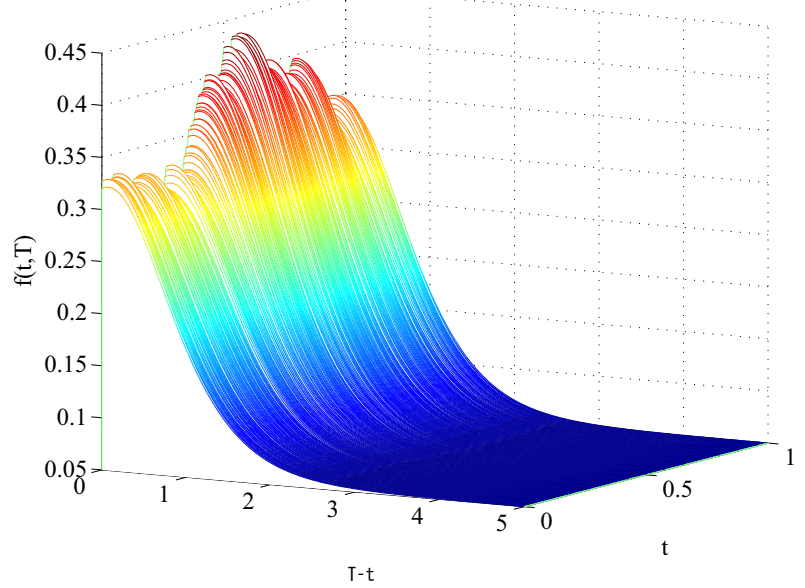

Fig. 3. A sample path of the forward rate given by (2) with $W_{0}=$ $8, c=0.1$.

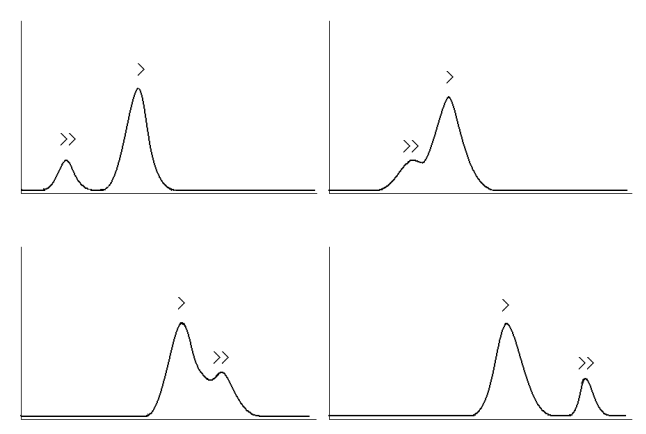

Fig. 4. Solitons.

the limits at $x \rightarrow \pm \infty$. If we require the inequality to be strict, then the graph $x \mapsto u(t, x)$ is hump-shaped. Note that solitons of this definition are stable under summation, multiplication, and differentiations. Note that the forward rate (2) in the previous section is a soliton in $T$ or $x=T-t$ in this sense.

\section{Affine term structure as multi-soliton}

We generalize the observation made in Section 2 . Let $W=\left(W^{1}, \ldots, W^{n}\right)$ be an $n$-dimensional Brownian motion starting at $x=\left(x^{1}, \ldots, x^{n}\right) \in \mathbf{R}^{n}$, defined on a filtered probability space $\left(\Omega, \mathcal{F}, P,\left\{\mathcal{F}_{t}\right\}\right)$, $\Lambda=\operatorname{diag}\left(\lambda_{1}, \lambda_{2}, \ldots, \lambda_{n}\right)$ with for each $\lambda_{i} \in \mathbf{R}(i=$ $1,2, \ldots, n)$, and $C \in M(n)$ be a positive definite matrix.

$$
\begin{aligned}
& \text { Let } \\
& \begin{aligned}
P(t, T):=e^{\left\langle C W_{t}, W_{t}\right\rangle} \\
\quad \times \mathbf{E}\left[e^{-\frac{1}{2} \int_{t}^{T}\left|\Lambda W_{s}\right|^{2} d s-\left\langle C W_{T}, W_{T}\right\rangle} \mid W_{t}\right] .
\end{aligned}
\end{aligned}
$$

Then $\{P(\cdot, T)\}$ defines an arbitrage-free bond market with

$$
\pi_{t}=e^{-\frac{1}{2} \int_{0}^{t}\left|\Lambda W_{s}\right|^{2} d s-\left\langle C W_{t}, W_{t}\right\rangle},
$$

being a state price density.

Proposition 1 Under the model (4), the forward rate $i s$ an $n$-soliton; a rational function in $e^{ \pm(T-t) \lambda_{i}}(i=$ $1,2, \ldots, n)$, of degree at most $2 n$ for any state $W_{t}$. 
Proof Let

$$
\begin{aligned}
& K(t)=-\cosh (t \Lambda) C-\frac{1}{2} \Lambda \sinh (t \Lambda), \\
& L(t)=2 \sinh (t \Lambda) \Lambda^{-1} C+\cosh (t \Lambda),
\end{aligned}
$$

and

$$
H(t)=K(t) \cdot L(t)^{-1}
$$

Note that

$$
K^{\prime}(t)=-\frac{1}{2} \Lambda^{2} L(t)
$$

and

$$
L^{\prime}(t)=-2 K(t)
$$

We will show that

$$
P(t, T)=(\operatorname{det}(L(T-t)))^{-\frac{1}{2}} e^{\left\langle(H(T-t)+C) W_{t}, W_{t}\right\rangle} .
$$

By the Feynman-Kac formula,

$$
u(t, x):=\mathbf{E}\left[e^{-\frac{1}{2} \int_{0}^{t}\left|\Lambda W_{s}\right|^{2} d s-\left\langle C W_{t}, W_{t}\right\rangle} \mid W_{0}=x\right],
$$

where $x=\left(x^{1}, \ldots, x^{n}\right)$, satisfies the following differential equation:

$$
\left\{\begin{array}{l}
\frac{\partial u}{\partial t}=\frac{1}{2} \Delta u-\frac{1}{2}\left\langle\Lambda^{2} x, x\right\rangle u \\
u(0, x)=e^{-\langle C x, x\rangle}
\end{array}\right.
$$

where $\Delta$ is the Laplacian. Note that

$$
P(t, T)=e^{\left\langle C W_{t}, W_{t}\right\rangle} u\left(T-t, W_{t}\right) .
$$

It is well-recognized that the solution $u$ to $(10)$ is expressed by

$$
e^{H_{0}(t)+\langle H(t) x, x\rangle}
$$

where $H$ is a symmetric-matrix valued differentiable function satisfying

$$
\frac{d H}{d t}(t)=2 H(t)^{2}-\frac{1}{2} \Lambda^{2}, \quad H(0)=-C,
$$

and $H_{0}$ is given by

$$
\frac{d H_{0}}{d t}(t)=\operatorname{tr} H(t), \quad H_{0}(0)=0 .
$$

Now we see that $H$ given by (5) and (6) is the unique solution to (13). In fact, by (7) and (8), we have

$$
\begin{aligned}
H^{\prime} & =\left(K L^{-1}\right)^{\prime}=-K L^{-1} L^{\prime} L^{-1}+K^{\prime} L^{-1} \\
& =2\left(K L^{-1}\right)^{2}-\frac{1}{2} \Lambda^{2}=2 H^{2}-\frac{1}{2} \Lambda^{2}
\end{aligned}
$$

and also $L(0)=I$ and $K(0)=-C$, which imply $H(0)=$ $-C$.

Further, by (14),

$$
\begin{aligned}
e^{H_{0}(t)} & =e^{\operatorname{tr}\left(-\frac{1}{2} \int_{0}^{t} L^{\prime}(s) L(s)^{-1} d s\right)} \\
& =\operatorname{det}\left(e^{-\frac{1}{2} \int_{0}^{t} L^{\prime}(s) L(s)^{-1} d s}\right) \\
& =\left(\operatorname{det}\left(e^{\int_{0}^{t} L^{\prime}(s) L(s)^{-1} d s}\right)\right)^{-\frac{1}{2}} \\
& =(\operatorname{det} L)^{-\frac{1}{2}}
\end{aligned}
$$

The last line needs some more lines of explanations, which we omit here.

Thus we have confirmed (9), at the same time (11) with (12), by which we have

$$
f(t, T)=-\frac{\partial}{\partial T} H_{0}(T-t)+\frac{\partial}{\partial T}\left\langle H(T-t) W_{t}, W_{t}\right\rangle .
$$

Then, by substituting (13) and (14), we get

$$
\begin{aligned}
f(t, T)=- & \operatorname{tr} H(T-t) \\
& -\frac{1}{2}\left\langle\left(4 H(T-t)^{2}-\Lambda^{2}\right) W_{t}, W_{t}\right\rangle .
\end{aligned}
$$

We note that the $(i, j)$-th entries $k_{i j}$ and $l_{i j}$ of $K(t)$ and $L(t)$ are given by

$$
k_{i j}=-\cosh \left(t \lambda_{i}\right) c_{i j}-\frac{1}{2} \delta_{i j} \sinh \left(t \lambda_{i}\right),
$$

and

$$
l_{i j}=2 \sinh \left(t \lambda_{i}\right) \lambda_{i}^{-1} c_{i j}+\delta_{i j} \cosh \left(t \lambda_{i}\right),
$$

and thus they are polynomials in $e^{ \pm t \lambda_{i}}$. Since

$$
H(t)=K(t) L(t)^{-1}=K(t) \tilde{L}(t)(\operatorname{det}(L(t)))^{-1},
$$

where $\tilde{L}(t)$ is the cofactor matrix of $L(t)$, we see that each entry of $H(t)$ is a rational function in $e^{ \pm t \lambda_{i}}(i=$ $1, \ldots, n)$, with degree $n$. Hence, by the expression (15), we have the assertion.

(QED)

Remark 2 It is known that the forward rates stay positive if $\pi$ is a strict supermartingale. In fact, for $T_{1}>T_{2}$ we have

$$
\mathbf{E}\left[\pi_{T_{1}} \mid \mathcal{F}_{t}\right]<\mathbf{E}\left[\pi_{T_{2}} \mid \mathcal{F}_{t}\right]
$$

by the supermartingale property of $\pi$, and the formula reads

$$
P\left(t, T_{1}\right)=\frac{\mathbf{E}\left[\pi_{T_{1}} \mid \mathcal{F}_{t}\right]}{\pi_{t}} \leq \frac{\mathbf{E}\left[\pi_{T_{2}} \mid \mathcal{F}_{t}\right]}{\pi_{t}}=P\left(t, T_{2}\right),
$$

meaning that $P(t, \cdot)$ and hence $\log P(t, \cdot)$ is decreasing. This in turn implies that $f(t, T)=-\partial_{T} \log P(t, T)$ is positive.

We give a sufficient condition that ensures the positivity. Since

$$
\begin{aligned}
d \pi_{t}= & \pi_{t}\left(-d\left\langle C W_{t}, W_{t}\right\rangle-\frac{1}{2}\left|\Lambda W_{t}\right|^{2} d t+\frac{1}{2} d\left[\left\langle C W_{t}, W_{t}\right\rangle\right]_{t}\right) \\
=- & 2\left\langle C W_{t}, d W_{t}\right\rangle-\operatorname{tr} C d t \\
& \quad-\frac{1}{2}\left|\Lambda W_{t}\right|^{2} d t+\frac{2^{2}}{2}\left|C W_{t}\right|^{2} d t
\end{aligned}
$$

we see that $\pi$ is a supermartingale, and hence the forward rates stay positive, if

$$
\Lambda^{2}-4 C^{2}>0
$$

since $C>0$ is already assumed.

\section{Remarks on a relation with $\mathrm{KdV}$ equa- tion}

Let $\tilde{f}(t, T):=f\left(\frac{c^{2}}{2^{4}} t, \frac{1}{2^{2}} T\right)$. Then, we have

$$
\tilde{f}(t, T)=\frac{c}{2^{3}} \tanh \left(\frac{1}{2}\left(\frac{c}{2} T-\frac{c^{3}}{2^{3}} t\right)\right)
$$




$$
\begin{aligned}
& +\frac{c^{2}\left|W_{t}\right|^{2}}{2^{3}} \operatorname{sech}^{2}\left(\frac{1}{2}\left(\frac{c}{2} T-\frac{c^{3}}{2^{3}} t\right)\right) \\
= & : v(t, T)+\left|W_{t}\right|^{2} u(t, T) .
\end{aligned}
$$

By this scale change, the functions $u$ and $v$ satisfy $4 \frac{\partial v}{\partial T}=$ $u$ and

$$
\frac{\partial u}{\partial t}=-6 u \frac{\partial u}{\partial T}-\frac{\partial^{3} u}{\partial T^{3}}
$$

Eq. (16) is known as the Korteweg-de Vries equation (KdV equation for short), which describes waves on shallow water surfaces. The KdV equation is mathematically as well as physically quite important in that there are many infinite dimensional symmetries which allow it to have great many explicit solutions including elliptic ones, rational ones, and most importantly in our context, soliton ones.

The relation has been extensively studied, especially by N. Ikeda and S. Taniguchi [6-10]. An extended relation to $\mathrm{KP}$ solitons using stochastic areas is given in $[11]$.

\section{Concluding remark}

We have pointed out that the forward rates of some (but actually almost all) affine term structures are multisolitons. This observation may give new insights to fitting or calibrating of affine term structures.

\section{Acknowledgments}

This work was supported by JSPS KAKENHI Grant Numbers 23330109, 24340022, 23654056 and 25285102. The authors thank Nobutaka Shimizu for providing the figures.

\section{References}

[1] N. L. Liu, A comparative study of principal component analysis on term structure of interest rates, JSIAM letters, 2 (2010), 57-60.

[2] N. L. Liu and M. E. Mancino, Fourier estimation method applied to forward interest rates, JSIAM Letters, 4 (2012), 17 20.

[3] N. L. Liu, I. Shin and Y. Yasuda, Principal component analysis applied to forward rates I: experiments with empirical data, in: Proc. of the 43rd ISCIE International Symposium on Stochastic Systems Theory and Its Application, pp. 235$241,2012$.

[4] D. Duffie, Dynamic Asset Pricing Theory, 3rd eds., Princeton University Press, N. J., 2001.

[5] R. Hirota, The Direct Method in Soliton Theory, Cambridge Tracts in Mathematics, Vol. 155, Cambridge University Press, Cambridge, 2004.

[6] N. Ikeda and S. Taniguchi, Quadratic Wiener functionals, Kalman-Bucy filters, and the KdV equation, Adv. Stud. Pure Math., 41 (2004), 167-187.

[7] S. Taniguchi, On Wiener functionals of order 2 associated with soliton solutions of the KdV equation, J. Funct. Anal., 216 (2004), 212-229.

[8] S. Taniguchi, Brownian sheet and reflectionless potentials, Stochastic Process. Appl., 116 (2006), 293-309.

[9] S. Taniguchi, Stochastic analysis and the KdV equation, Contemp. Math., 429 (2007), 245-256.

[10] N. Ikeda and S. Taniguchi, The Itô-Nisio theorem, quadratic Wiener functionals, and 1-solitons, Stochastic Process. Appl., 120 (2010), 605-621.

[11] H. Aihara, J. Akahori, H. Fujii and Y. Nitta, Tau functions of
KP solitons realized in Wiener space, Bulletin of the London Mathematical Society, 2013, DOI: 10.1112/blms/bdt056. 